\title{
An EEG investigation on planning human-robot handover tasks
}

\author{
$1^{\text {st }}$ Sara Cooper ${ }^{1}$ \\ School of Engineering and Physical \\ Science, Sensors, Signals and Systems \\ Heriot-Watt University \\ Edinburgh, UK \\ Email: sara.cooper@pal-robotics.com \\ $2^{\text {nd }}$ Stuart Gow ${ }^{2}$ \\ Psychology, Faculty of Natural Sciences \\ University of Stirling \\ Stirling, UK \\ Email: sag00097@students.stir.ac.uk
}

\author{
$3^{\text {rd }}$ Samuel F.P. Fensome \\ University of Stirling \\ Stirling, UK \\ Email: s.f.bennett@stir.ac.uk \\ $4^{\text {th }}$ Mauro Dragone \\ School of Engineering and Physical \\ Science, Sensors, Signals and Systems \\ Heriot-Watt University \\ Edinburgh, UK \\ Email: m.dragone@hw.ac.uk
}

Psychology, Faculty of Natural Sciences Psychology, Faculty of Natural Sciences

\begin{abstract}
Human-robot joint action is a key requirement in many advanced robotic applications where robots are not only expected to work alongside humans but also collaborate with them in the performance of physical tasks. Robots are already programmed to model and predict human actions in order to ensure smooth collaboration and overall task efficiency. However, little is known on how humans represent and account for robot's actions as part of their own plans. This paper presents a first joint psychological and HRI user study designed to answer this question in the context of human-robot handover scenarios. Our analysis showed that the participants had a positive userexperience of the interaction and adopted gaze patterns similar to a large extent to the ones in human-to-human handover tasks. The EEG analysis suggests that, compared to solo action, the human participants were at a state of higher motor readiness when they prepared to hand over the object to the robot either because they represented the robot's action in advance or alternatively that they anticipated that passing the object to the robot would be a more effortful action, thus highlighting the increased demands in planning a human-to-robot interaction. Our findings highlight the value of gaze as a positive method of non-verbal communication in HRI and provides new insights in the neural mechanisms that allows a person to plan an effective interaction with a robot.
\end{abstract}

Index Terms-Human-Robot Interaction, EEG, handover, joint-action

\section{INTRODUCTION}

From industrial robotic systems operating in the same physical space as people, to domestic robot companions and assistive robots, who are expected to play an increasingly bigger role in physical aspects of care, the use of robots for physical tasks will only increase in the future, and with

*Research output of Sara's ${ }^{1}$ MSc in Robotics at Heriot-Watt University and Stuart's ${ }^{2}$ MSc in Psychology at Stirling University in 2018-2019 it, the need to collaborate and perform joint actions with humans. This may be for the purpose of helping in assembly and other manufacturing tasks, unloading weights or handing over objects at home. Researchers have long recognised the importance of establishing and maintain a mutual understanding between humans and robots during joint action. All the situations above require coordination from both agents in order to reach a joint goal and, in doing so, observe and predict each other's actions. A handover, consisting of the transfer of an object from giver to receiver, is possibly the simplest kind of joint action that makes it possible to ensure physical collaboration. Much work has already been done in developing human-robot handovers, having robots predict human movement, ensuring grasping accuracy and task efficiency. A key objective of Human-Robot Interaction (HRI) related work is to increase robot acceptability. To do so, robots must be shown to be useful, predictable and safe. During joint actions, how a robot expresses its intention may help the user understand what they are going to do, hence improving joint action planning and performance [1]. Also, understanding what takes place in the mind of the human during a handover when the co-actor is a robot, should suggest future trends in robot design. We know that humans mentally represent their partner's actions during joint tasks in order to improve coordination and taskefficiency, and to do so, they form internal representations of their own part of the joint task in addition to their co-actors' parts of the task [2] [3]. But how do humans feel about the interaction with a robot? Do we also consider robots as joint action partners, and co-represent their actions?

Related works have focused on different phases of a handover such as approach, intention transmission and the handover itself [4], in order to outline preferred types of robot 
behaviour in each case, and investigate specific aspects of the handover, such as grip force, arm trajectory analysis and velocity patterns. While recent analysis of user preferences of non-verbal cues such as gaze/head pose orientation or gestures has gained importance as well, these aspects of humanrobot interaction are usually deduced and assessed through video analysis or through questionnaires. Electrophysiological evidence as to whether adjusting these parameters really helps humans predict the robot's action or regarding them as joint action partners is still lacking and could be an invaluable tool especially to inform the development of user-friendly robot behaviours.

\section{METHODS}

\section{A. Participants}

16 participants $(8$ female and 8 male, age $=26.6 \pm 9.0$ years). All participants gave their informed consent after full explanation of the study. A short familiarity survey showed that $81.5 \%$ of the participants had had previous interactions with robots. Six participants were excluded from the EEG analysis due to poor quality signal, primarily because of excessive movements during the action planning phase.

\section{B. Experimental set-up and procedure}

A Tiago ${ }^{1}$ humanoid manipulator robot (PAL Robotics) and a human participant were seated at opposite sides of a rectangular table. An empty box of cereals was placed halfway between the participants, and an LCD projector was mounted on a tripod extension arm, placed next to the table, in order to project visual stimuli on both sides of the object. Action onsets were recorded via a keypad, positioned in front of the participant's right hand. The participant was instructed to continually press a designated key with his/her index finger and only release it for the performance of an action. Each trial started with the display of a black fixation cross for 1000 $\mathrm{ms}$, after which an informative Cue (diamond, circle or square) stimulus was displayed for $250 \mathrm{~ms}$ and indicated the type of action that the participant was required to prepare, namely:

1) Solo Action: the human releases the key, reaches, grasps, raises and holds the object for approximately 5-6 sec and finally lowers it to the starting position.

2) Joint Action - Human Receiver: Tiago starts from a predefined starting position as it looks down. It first looks at the object and moves the gripper to the pregrasp position, before grasping and raising it. The human then receives the object and places it back, as Tiago withdraws the gripper.

3) Joint action - Human Giver: the human releases the key, reaches, grasps, raises and holds the object until Tiago receives it and places it to the starting position.

The participants were instructed to withheld their action until the display on an imperative Go stimulus (a green cross), which prompted to perform the prepared action.

Figure 2 shows an example of an object being transferred from the participant to Tiago.

\footnotetext{
${ }^{1} \mathrm{http}: / /$ tiago.pal-robotics.com/
}

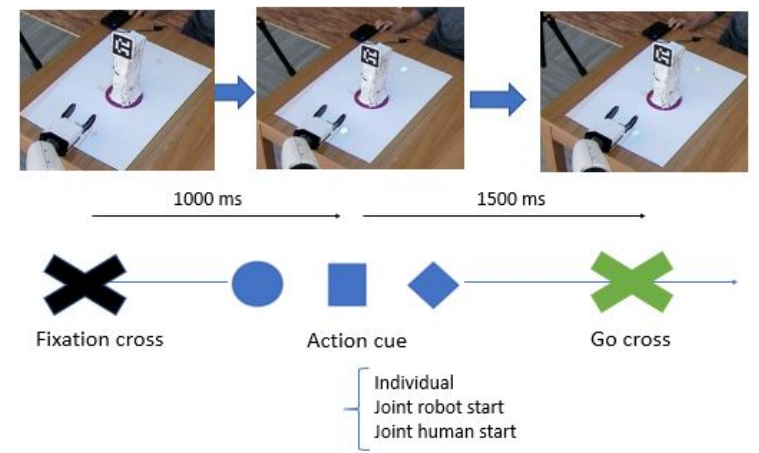

Fig. 1: Cue timeline 1) Fixation cross, 2) Cue indicating the type of action to be prepared, 3) Go stimulus prompting action initiation

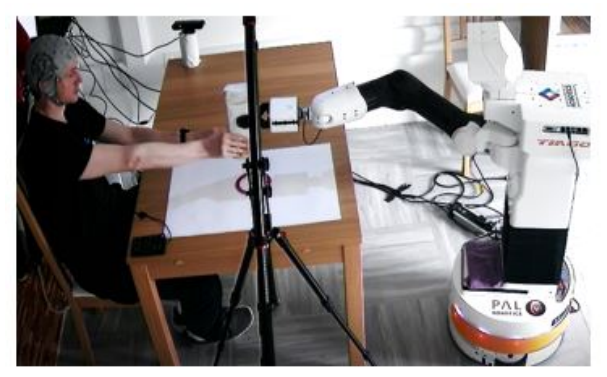

Fig. 2: Object transfer in human-to-robot handover

The experiment was divided into a practice block and 5 blocks of 21 trials, each lasting between 5 and 8 minutes, with an equal number of random variations of action types. At the end of the experiment participants were required to answer a survey regarding robot acceptance and impression of the handover, using a 5-point Likert scale [5] [6].

\section{EEG Data acquisition, processing and analysis}

EEG data was recorded with a Curry v7 EEG acquisition software using a purposefully positioned 64- electrode nylon cap (Quik-Cap) via a SynAmps RT64 amplifier (EEG software and equipment by Compumedics Neuroscan). The electrodes were arranged according to the extended 10/20 system placement standard. Vertical and horizontal eye movement were monitored via bipolar par of electrodes placed positioned above and below the middle of the left eye and lateral to each eye, respectively. Electrode impedance was kept below $10 \mathrm{k} \Omega$. EEG signals were amplified with a band-pass filter of $0-400 \mathrm{~Hz}$ and sampled at $1000 \mathrm{~Hz}$.

EEG data processing was performed using the BrainVision Analyzer software (BVA v.2.1 Brain Products). First, the EEG data were re-referenced to the mean signal from both mastoids. The data were filtered with a low cut-off filter of $0.05 \mathrm{~Hz}$ (24 dB/octave) and a high cut-off filter of $30 \mathrm{~Hz}(24$ $\mathrm{dB} /$ octave) and a notch filter at $50 \mathrm{~Hz}$ to suppress power line interference. Ocular Correction was performed on continuous data using the Gratton \& Coles algorithm [7]. Then, the data were segmented into epochs from $300 \mathrm{~ms}$ before Cue onset 
until 1000ms Go onset. Semi-automatic artefact rejection was performed in order to remove remaining non-EEG related artefacts. Averages were created separately for each condition and each participant. The last $200 \mathrm{~ms}$ before Cue onset was set as the baseline period.

EEG analysis was focused on the Contingent Negative Variation (CNV), a slow-rising Event Related Potential of negative polarity that develops between an informative Cue and an Imperative Go stimulus and peaks at approximately the time of a planned response [8]. The CNV is related to stimulus anticipation and movement planning [9] and it reflects preparation and organization of a motor program that may involve multiple actions performed sequentially or synchronously by a pair of individuals [2] [3].

\section{IMPLEMENTATION}

The robot was programmed using the Robotic Operating System (ROS) while the Psychoobox toolbox for Matlab [10] was used to specify the flow of the study and display the respective cues (e.g. random order of the queues for different trials and blocks, for each participant), send triggers to EEG, send/receive triggers from Tiago, and receive action onset data from the participant (keypad release). Communication between the two systems is achieved through a wireless link, using the Matlab Robotics System Toolbox ${ }^{2}$. The same method is also used to let Tiago know what action it needs to perform.

The object handover was designed by taking into account key findings from human-robot action research..

Given the large number or repetitions necessary for good EEG signal-to-noise ratio, robustness is a key requirement for the handover. In order to enrich the interaction, aspects outlined in [4] have been taken into consideration, such as handover forcefulness during the object exchange, aggressiveness when approaching object, smooth and humanlike movements, and short interaction times.

The object to be grasped in our experiment is tall enough to ensure a safe handover. Specifically, Tiago is programmed to grasp the object from the top when taking it from the table, and from the bottom when taking it from the human, thus increasing predictability and safety. Due to the importance of grasping accuracy, object recognition is ensured through an Aruco marker ${ }^{3}$, placed on the top lateral side of the object, visible in Figure 3. Such fiducial markers offer high detection rates and are very easy to use [11].

Rather than using a grasp planner, we set three possible grasp points (relative to the Aruco marker), respectively prioritising picking from the top, for robot-to-human handover, and the opposite for human-to-robot. A constant and highly repetitive motion was achieved by using the MoveIt! framework [12] to move the arm to the goal from a predefined resting position. To improve the interaction, we prioritised achieving a minimum jerk trajectory profile [13] [14] [15] reported to be most humanlike during handovers. To do this,

\footnotetext{
${ }^{2}$ https://nl.mathworks.com/products/robotics.html

${ }^{3}$ http://wiki.ros.org/aruco
}

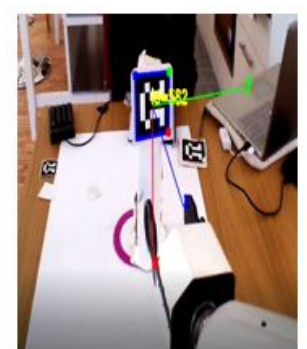

(a) Tracking of the aruco marker

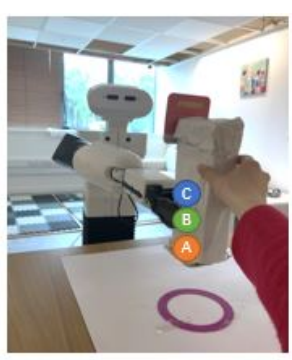

(b) Grasp points for safe human-to-robot handover
Fig. 3: Object grasping methodology

time parameterization was applied to all 7 joints of Tiago's arm, by also minimising planning and execution time (Figure 4).

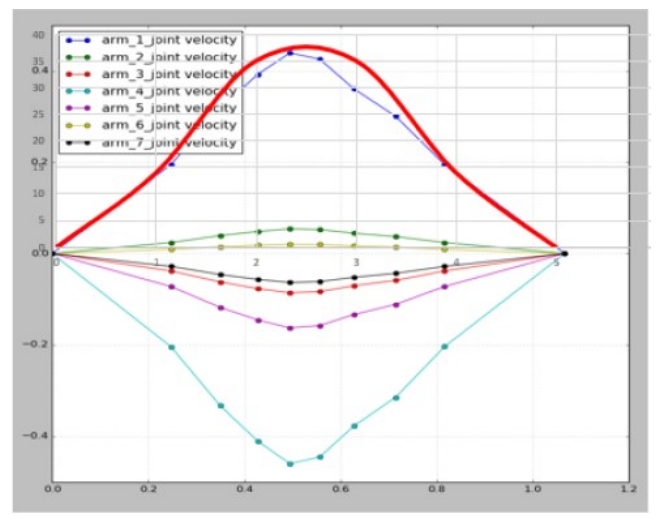

Fig. 4: Minimum-jerk trajectory profile implementation for all 7 joints of Tiago's arm, where the red line indicates the ideal parabolic shape for a sample joint

Safe object transfer is essential in order to open and close the gripper at suitable times and prevent the object from slipping [16]. To model the object exchange, a simplified version of the human-inspired gripper proposed by [17] was implemented for robot-to-human handovers, which was also reused by [16] and [18]. Specifically, Tiago is programmed to start to release the object once a specific threshold of pull force is surpassed, opening proportionally to the force applied.

For human-to-robot handover, Tiago monitors the alignment of gripper and goal and ensures stability of the object before approaching it to reduce aggressiveness in the approach (speed less than $0.01 \mathrm{~m} / \mathrm{s}$ ). Stability (e.g. object velocity) is also measured in robot-to-human, and, in the event the speed is higher than the threshold during the exchange, Tiago will regrasp the object so as to prevent handover failure [19].

Having Tiago behave in a humanlike way is expected to improve the user's impression and increase the chances of being regarded as a joint action partner. One way to achieve this is by using gaze cues.

Communication through gaze cues can help achieve mutual awareness [20], and thus increase handover safety and 
coordination [21] [7] [22]. Humans will follow the robot's gaze direction in order to predict the handover location and/or intention [20]. Similarly, a humanlike robot gaze pattern, such as focusing on the co-actor or other areas of interest, is predicted to improve the quality of interaction and lead to increased task efficiency [16]. In particular, Moon et al. [16] suggested that humans show turn taking or shared attention gaze patterns when giving an object, which involves gazing at the projected handover location and quickly glancing at the co-actor just before completing the trajectory.

Taking this into consideration, for robot-to-human handover, Tiago shows turn-taking gaze behaviour, by looking up at the the projected handover location and glancing up at the participant before reaching the object offer location, looking at the object otherwise as it is moved. In human-to-robot handover, Tiago first looks up to the human, attempting to establish eye contact as it starts to move the gripper, and later tracks the object as it is offered by the human.

Figure 5 and 6 show the summary of the procedure and the overall components of the user study. The behavioural control laptop, with Matlab, projects the stimuli on the table and sends triggers to EEG at different stages of the handover, using a keypad to record the participant's action onset. The Tiago control laptop contains all the ROS nodes, and connects to the former PC so that the robot knows which action it needs to do, and also lets the EEG know when Tiago is moving or not, by using ROS topics. The EEG acquisition laptop is connected to the EEG cap and amplifier and visualises and stores EEG data using Curry software and receives the timestamps from Matlab.

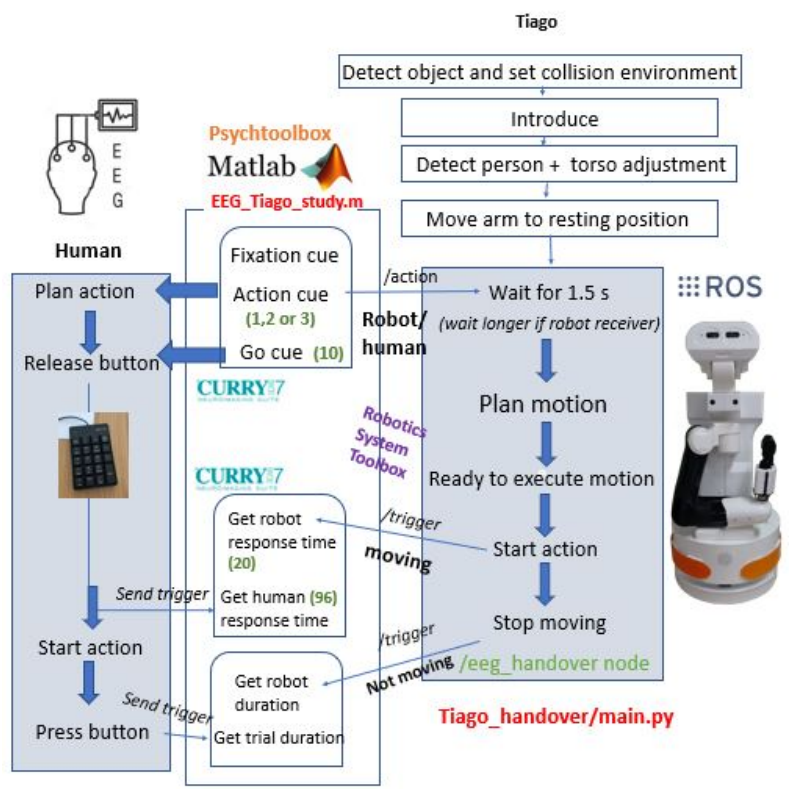

Fig. 5: Software architecture for interconnection of EEG, Tiago and cue presentation and data collection

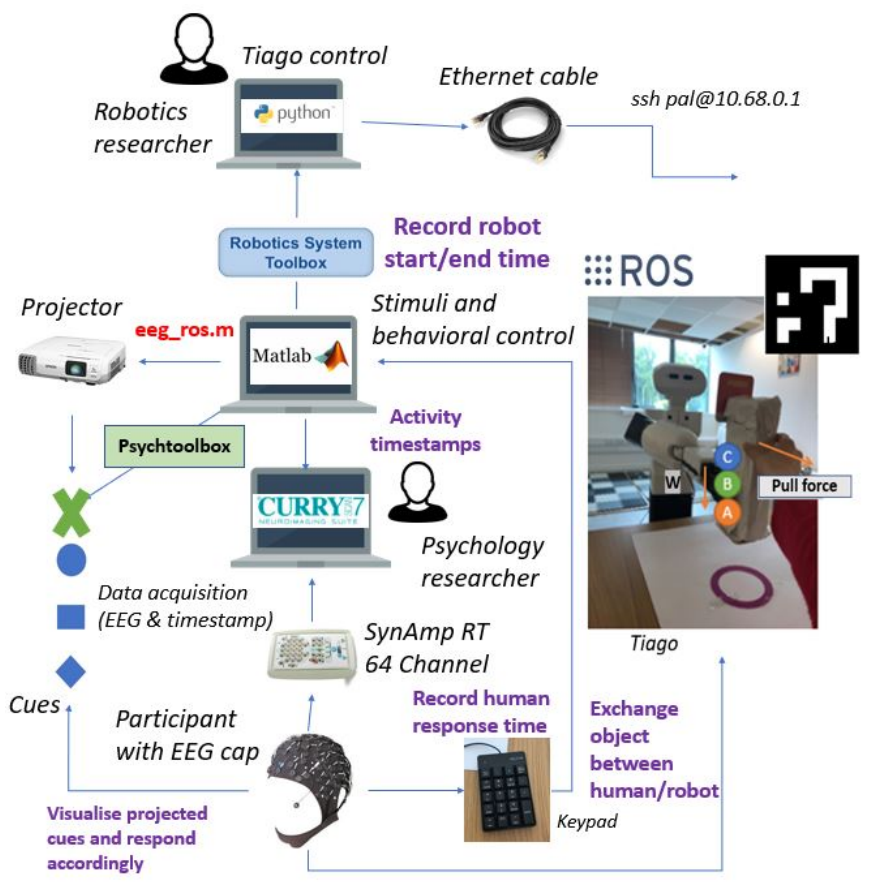

Fig. 6: Overall set-up infrastructure

\section{RESULTS}

\section{A. Robot acceptability analysis}

In order to measure robot acceptability, we used the results from the Qualtrics survey (Qualtrics, Provo, UT), which consists of positive and negative statements regarding the interaction. A 3.93/5 and 1.49/5 mark respectively for each suggests a generally favourable experience. A Mann-Whitney test showed significant differences in user impressions between those with robotics background $(\mathrm{N}=8, \mathrm{M}=4.17, \mathrm{SD}=0.89)$ and those without $(\mathrm{N}=8, \mathrm{M}=3.66, \mathrm{SD}=0.9)$. Overall, participants did not feel intimidated by the robot and believed they could quickly learn to perform joint tasks with the robot. Many pointed out that the handover was slow and did not think the robot's movements were humanlike, most likely because of the constant handover repetitions and lack of unpredictability.

\section{B. Handover timing and adaptation analysis}

Average transfer time of the object from Tiago to human was 1.11 seconds $(\mathrm{SD}=.89)$, and predictably participants recorded a high feeling of safety during object transfer (3.44/5 rate on object safety, higher rate in robot-to-human), similar to [17]'s. The overall timing of the handover was 18.49 seconds for human-to-robot, and 16 seconds for robot-to-human, while between humans takes less than 2 seconds. For this reason, we were interested to see whether human idle time - defined as the time from when the person releases the keypad after the GO signal until it takes the object - would be reduced as participants learned to use the robot [23] [16]. As expected, conducting a one-way ANOVA with Bonferroni correction ( $p ; 0.041$ ) over the first two blocks demonstrates that the participants object reaching time decreases as they do more 
handovers $-3.85 \mathrm{~s}$ average on the first block $(\mathrm{SD}=4.282)$ to $1.245 \mathrm{~s}$ on the second block $(\mathrm{SD}=1.48)$. This suggests that participants find it easy to adapt by observing and predicting Tiago's movements.

\section{Gaze analysis}

To evaluate human gaze behaviour, only the first 3 trials were considered from the first block of each participant, before they became too used to the robot [24]. In total 33 trials involving 11 participants were used for the analysis, as in other cases the object would occlude the person's face. A video annotation tool was used to evaluate the gaze pattern, due to the difficulties in maintaining a camera close enough to the person without disturbing the task. Overall, the gaze findings analysis shows that $80 \%$ of the participants look at Tiago at least once during the handover, which suggests that they regarded the interaction with Tiago in a similar way as a joint action with a human agent [21] [25].

In robot-to-human handovers, participants usually looked at Tiago when it began to reach for the object (18/32 of the trials), even if Tiago was only looking at the object. Moreover, they looked at Tiago when they were pulling from the object (Figure 7), instead of when Tiago was looking at them. This was most likely due to the decreased motion speed, and having no need to particularly infer the handover location. In between they would focus on the areas of interest (gripper/object), as reported by [16] [25].

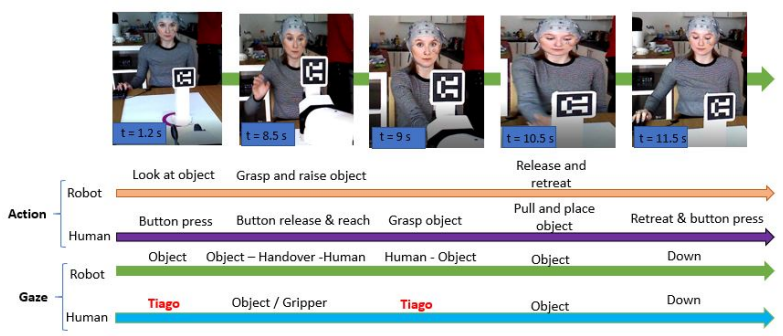

Fig. 7: Typical robot-to-human gaze pattern behavior

In human-to-robot handovers, participants looked at Tiago almost every time they offered the object (28/34 of the trials). They would not reciprocate Tiago's gaze contact at the beginning of the task, but instead focussed their gaze on the object (Figure 8), most likely because there was no need to communicate their intention to Tiago due to the presence of projection cues. A study with no such cues could provide further insight into human gaze response.

\section{EEG results}

Participants' behaviour showed a great inconsistency in the way they received the object from Tiago. Four out of ten participants released the button $600 \mathrm{~ms}$ after Go onset and hovered over it until Tiago offered the object, whereas six participants waited for an average time of $7,500 \mathrm{~ms}$ before releasing the button. For this reason, we have excluded the 'Receiving' condition from the EEG analysis (Figure 9).

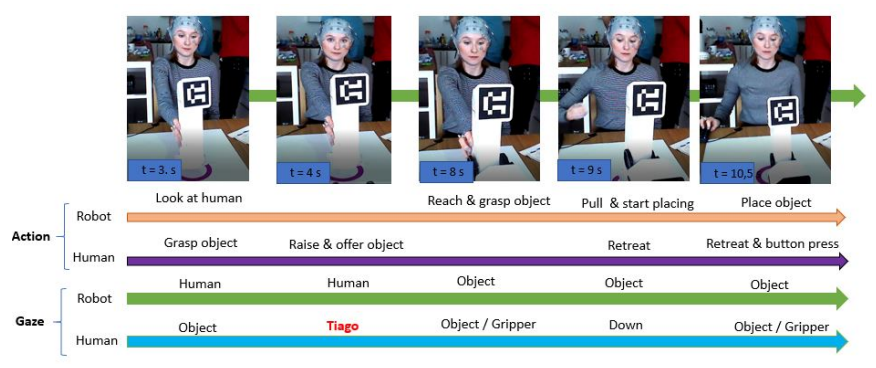

Fig. 8: Typical human-to-robot participant gaze pattern behavior

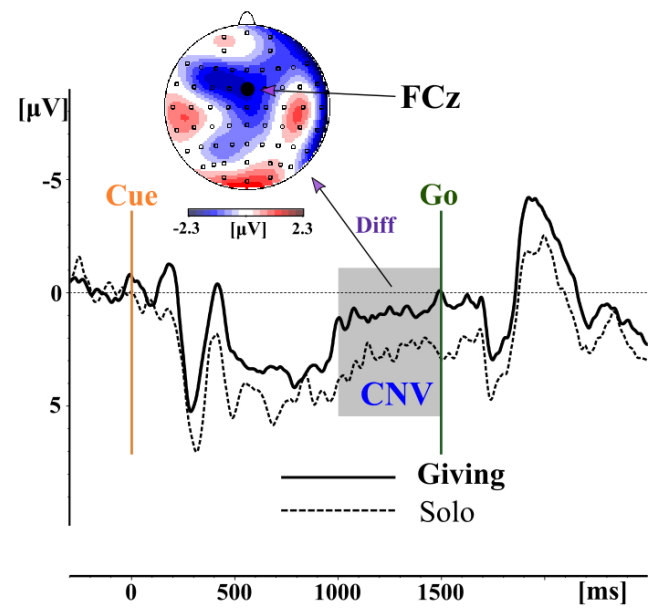

Fig. 9: ERP waveforms from electrode $\mathrm{FCz}$ and voltage scalp topography of the difference between Passing the Object and Solo action during the last 500 before the Go stimulus onset

The CNV was quantified as the mean amplitude of the last $500 \mathrm{~ms}$ before Go onset from electrode $\mathrm{FCz}$, which is placed over the Supplementary Motor Area, a brain region heavily involved in planning and coordination of action sequences [26]. A paired t-test showed that the CNV amplitude was significantly larger $(t(9)=2.7, p=.024)$ when the participants planned to pass the object compared to acting individually. This suggests that the participants were at a state of higher motor readiness when they planned to interact with Tiago well before action initiation. This may be viewed as an indication that they represented Tiago's action in advance or alternatively that they anticipated that passing the object would be a more effortful action. This is in contrast with a previous EEG study on human joint action planning, in which the CNV was of similar amplitude before passing an object and acting individually [2], thus, highlighting the different demands in planning a human-to-robot interaction compared to humanhuman interaction.

\section{CONCLUSIONS}

We have illustrated a combined HRI and psychology userstudy framework providing initial EEG evidence of joint action planning during bi-directional handover tasks. The main 
conclusion is that planning to hand over an object to a robot is more cognitively demanding than planning a similar action directed to another human.

Our findings show that the interaction was regarded as being highly positive. Moreover, specific focus was paid on the participant's gaze pattern. Reciprocal gaze was observed at the beginning of the robot-to-human handover. In human-torobot, there was a great tendency to look at the robot during the object exchange, suggesting that having the robot gaze at the human during that time can be beneficial. Lastly, humans were seen to adapt to robot movement and speed as they repeated the action, although due to the high predictability of the actions further work would be needed to validate this.

The lack of natural handover scenario (repetitive, keypad and cues) and the inclusion of a practice block may have resulted in the over-familiarization of participants with the task. Differences in the setup of similar human-to-human studies [2] make an accurate comparison difficult. While a robust and predictable handover was achieved to to ensure high success rate over trials, this may have resulted in making the robot too easy to predict. Analysis of EEG signals at different stages of the handover could shed information on the effect of action planning during task execution. A reactive handover controller could both increase handover speed and make the interaction slightly less predictable, which could help evaluate joint-action planning in robot-to-human handover as well. Gaze tracking systems would also make it easier to analyse and reciprocate human gaze and enrich the interaction. The sample size for the user-studies was also relatively small, especially as several cases had to be excluded for gaze analysis due to eye occlusion. A bigger sample size could provide stronger support for the hypothesis.

Our future studies will seek to address the above limitations in order to further our investigation on joint-action planning and understand its impact on effective human-robot collaboration, especially in assistive scenarios.

\section{ACKNOWLEDGMENT}

The work described in this paper was partially funded by the UK project ORCA Hub (UK Robotics and Artificial Intelligence Hub for Offshore Energy Asset Integrity Management), EPSRC Reference: EP/R026173/1.

\section{REFERENCES}

[1] B. et al., "Implicitly assisting humans to choose good grasps in robot to human handovers," in International Symposium on Experimental Robotics. Springer, 2016, pp. 341-354.

[2] D. Kourtis, N. Sebanz, and G. Knoblich, "Predictive representation of other people's actions in joint action planning: An eeg study," Social neuroscience, vol. 8, no. 1, pp. 31-42, 2013.

[3] D. Kourtis, G. Knoblich, M. Woźniak, and N. Sebanz, "Attention allocation and task representation during joint action planning," Journal of Cognitive Neuroscience, vol. 26, no. 10, pp. 2275-2286, 2014.

[4] K. Strabala, M. K. Lee, A. Dragan, J. Forlizzi, S. S. Srinivasa, M. Cakmak, and V. Micelli, "Toward seamless human-robot handovers," Journal of Human-Robot Interaction, vol. 2, no. 1, pp. 112-132, 2013.

[5] J. M. Beer, A. Prakash, T. L. Mitzner, and W. A. Rogers, "Understanding robot acceptance," Georgia Institute of Technology, Report, 2011
[6] M. Heerink, B. Kräse, V. Evers, and B. Wielinga, "Assessing acceptance of assistive social agent technology by older adults: the almere model," International journal of social robotics, vol. 2, no. 4, pp. 361-375, 2010.

[7] G. Gratton, M. G. Coles, and E. Donchin, "A new method for offline removal of ocular artifact," Electroencephalography and clinical neurophysiology, vol. 55 , no. 4, pp. 468-484, 1983.

[8] W. G. Walter, R. Cooper, V. Aldridge, W. McCallum, and A. Winter, "Contingent negative variation: an electric sign of sensori-motor association and expectancy in the human brain," nature, vol. 203, no. 4943, pp. 380-384, 1964.

[9] H. et al., "Preparing for action: inferences from cnv and lrp," Journal of psychophysiology, vol. 18, no. 2/3, pp. 77-88, 2004.

[10] D. H. Brainard, "The psychophysics toolbox," Spatial vision, vol. 10, no. 4, pp. 433-436, 1997.

[11] M. Ciocarlie, K. Hsiao, E. G. Jones, S. Chitta, R. B. Rusu, and I. A. Ǎžucan, "Towards reliable grasping and manipulation in household environments," in Experimental Robotics. Springer, 2010, Conference Proceedings, pp. 241-252.

[12] D. Coleman, I. Sucan, S. Chitta, and N. Correll, "Reducing the barrier to entry of complex robotic software: a moveit! case study," arXiv preprint arXiv: $1404.3785,2014$

[13] S. et al., "Experimental analysis of handing over," in Proceedings 4th IEEE international workshop on robot and human communication. IEEE, 1995, Conference Proceedings, pp. 53-58.

[14] M. Huber, M. Rickert, A. Knoll, T. Brandt, and S. Glasauer, "Humanrobot interaction in handing-over tasks," in RO-MAN 2008-The 17th IEEE International Symposium on Robot and Human Interactive Communication. IEEE, 2008, Conference Proceedings, pp. 107-112.

[15] U. Pattacini, F. Nori, L. Natale, G. Metta, and G. Sandini, "An experimental evaluation of a novel minimum-jerk cartesian controller for humanoid robots," in 2010 IEEE/RSJ international conference on intelligent robots and systems. IEEE, 2010, Conference Proceedings, pp. 1668-1674.

[16] A. Moon, D. M. Troniak, B. Gleeson, M. K. Pan, M. Zheng, B. A. Blumer, K. MacLean, and E. A. Croft, "Meet me where i'm gazing: how shared attention gaze affects human-robot handover timing," in Proceedings of the 2014 ACM/IEEE international conference on Humanrobot interaction. ACM, 2014, Conference Proceedings, pp. 334-341.

[17] W. P. Chan, C. A. Parker, H. M. Van Der Loos, and E. A. Croft, "A human-inspired object handover controller," The International Journal of Robotics Research, vol. 32, no. 8, pp. 971-983, 2013.

[18] K. et al., "Learning dynamic robot-to-human object handover from human feedback," arXiv preprint arXiv:1603.06390, 2016.

[19] M. S. Parastegari, "Modelling and control of object handover, a study in human-robot interaction," Thesis, 2018.

[20] C.-M. Huang, M. Cakmak, and B. Mutlu, "Adaptive coordination strategies for human-robot handovers," in Robotics: science and systems. Rome, Italy, 2015, Conference Proceedings.

[21] A. Curioni, G. Knoblich, and N. Sebanz, "Joint action in humans: A model for human-robot interactions," Humanoid Robotics: A Reference, pp. 1-19, 2016.

[22] M. Cakmak, S. S. Srinivasa, M. K. Lee, S. Kiesler, and J. Forlizzi, "Using spatial and temporal contrast for fluent robot-human handovers," in 2011 6th ACM/IEEE International Conference on HumanRobot Interaction (HRI). IEEE, 2011, pp. 489-496.

[23] M. Huber, M. Rickert, A. Knoll, T. Brandt, and S. Glasauer, "Humanrobot interaction in handing-over tasks," in RO-MAN 2008-The 17th IEEE International Symposium on Robot and Human Interactive Communication. IEEE, 2008, Conference Proceedings, pp. 107-112.

[24] M. Zheng, A. J. Moon, B. Gleeson, D. M. Troniak, M. K. Pan, B. A. Blumer, M. Q. Meng, and E. A. Croft, "Human behavioural responses to robot head gaze during robot-to-human handovers," in 2014 IEEE International Conference on Robotics and Biomimetics (ROBIO 2014). IEEE, 2014, Conference Proceedings, pp. 362-367.

[25] J. Pérez-Osorio, D. De Tommaso, E. Baykara, and A. Wykowska, "Joint action with icub: a successful adaptation of a paradigm of cognitive neuroscience in hri," in 2018 27th IEEE International Symposium on Robot and Human Interactive Communication (RO-MAN). IEEE, 2018, pp. $152-157$.

[26] R. Cui, A. Egkher, D. Huter, W. Lang, G. Lindinger, and L. Deecke, "High resolution spatiotemporal analysis of the contingent negative variation in simple or complex motor tasks and a non-motor task," Clinical Neurophysiology, vol. 111, no. 10, pp. 1847-1859, 2000. 